\title{
Grenzblicke
}

\section{Die Einführung von diagnoseorientierten Fallpauschalen in Deutschland}

\section{J. Dratva}

1 Textdokumentation der deutschen Bundesregierung «Neue, leistungsgerechte Vergütung der Krankenhäuser» 1. März 2002.

http://text.bundesregierung.de.

2 Bundesministerium für Gesundheit. Pressemitteilung Nr. 137. 14. Dezember 2001

- http://www.bmgesundheit.de.

- http://text.bundesregierung.de.

- http://bundesrat.de.

- http://www.bundesärztekammer.de.

- http://www.marburger-bund.de.

- http://www.dkgev.de.

- http://www.fischer-zim.ch.

- http://www.g-drg.de.
Am 1. März 2002 hat der deutsche Bundesrat (Länderkammer) als letzte Instanz dem diagnoseorientierten Fallpauschalengesetz zugestimmt. Die deutsche Bundesgesundheitsministerin Schmidt betont, «mit dem Fallpauschalengesetz sei der Weg zu einer leistungsgerechten Vergütung endlich frei» [1]. Es werden «die Rahmenbedingungen für ein modernes, Qualität, Transparenz und Wirtschaftlichkeit förderndes Vergütungssystem geschaffen» [2].

Für die Vertragspartner besteht endlich Rechtssicherheit. Ein Ende der Diskussionen und Verhandlungen ist jedoch noch nicht abzusehen. Trotz eines breiten Konsenses über die Notwendigkeit eines modernen Vergütungssystems für das stationäre und teilstationäre Gesundheitswesen besteht in Detailfragen eine grosse Meinungsdifferenz.

\section{Übereilt und fehlerhaft}

Hauptkritikpunkte der Bundesärztekammer Deutschlands sind u. a. die übereilte Einführung des Fallpauschalensystems, die weiterhin bestehende Budgetierung der Ausgaben und die unzureichenden Bestimmungen für eine wirklich leistungsbezogene Vergütung. Kritisiert wird auch die fehlende Berücksichtigung der demographisch-epidemiologischen Entwicklung und der zukünftigen medizinischen Innovationen sowie die Nichtbeachtung des tatsächlichen Bedarfs an medizinischen Fachkräften.

\section{Fallpauschalen statt Pflegesätze}

Das Fallpauschalengesetz sieht vor, dass die von den Krankenhäusern erbrachten Leistungen zukünftig nach Fallpauschalen für definierte Krankheitsfälle (sogenannte DRG - diagnose related groups) vergütet werden. Damit wird die bisherige Vergütung von Pflegesätzen pro Tag und Einzelleistungen grundlegend reformiert. Bei der Erstellung der deutschen DRG und der Kodierungsrichtlinien wurde auf das australische DRG-System (AR-DRG Version 4.1) zurückgegriffen. In einer Übergangsphase bis 2006 sollen die nötigen Anpassungen an die deutschen Verhält- nisse erfolgen. Die DRG-Fallpauschalen richten sich nach Diagnose-, Behandlungs- und Operationsschlüsseln. Bei den Diagnoseschlüsseln wird in Hauptdiagnose und Nebendiagnosen unterteilt. Die Nebendiagnosen erlauben, mögliche Komplikationen und somit den Schweregrad einer Erkrankung zu kodieren. Alter und Geschlecht werden ebenfalls kodiert. Die DRG gelten für alle Fachbereiche im stationären und teilstationären Gesundheitswesen mit Ausnahme einiger psychiatrischer Institutionen.

Die australischen DRG-Gruppen und Kostengewichte werden in einer Übergangszeit für die deutschen Berechnungen eingesetzt. Leider ist deren Eignung dazu aufgrund der so unterschiedlichen Strukturen der Gesundheitswesen und Bedürfnisse in Frage zu stellen. Die Anpassung, die voraussichtlich erhebliche Arbeitsprozesse mit sich bringen wird, ist in der auf 2 Jahre bemessenen Zeit nicht machbar. Eine kostengerechte Kodierung wird ab 2005 kaum möglich sein. Auch sollte ernsthaft über einen zusätzlichen pflegerischen Code nachgedacht werden, der die zunehmende pflegerische Arbeit und die Kosten dokumentiert. Diese erbrachte Leistung ist aus der geplanten medizinischen Kodierung nicht genügend $\mathrm{zu}$ ersehen. Es wird nicht $\mathrm{zu}$ vermeiden sein, dass einige Diagnosen und medizinische Behandlungen zum Anwendungszeitpunkt noch nicht kodiert sind oder nicht kodierbar sind. Hierfür sollten über das Jahr 2004 hinaus Einzelabrechnungen möglich bleiben.

\section{Ein zu straffer Zeitplan}

Das Gesetz tritt am Januar 2003 zunächst auf freiwilliger Basis in Kraft. Die obligate Einführung erfolgt zum 1. Januar 2004. Die Jahre 2003 und 2004 dienen als budgetneutrale Einführungsphase. Erst ab 2005 soll der finanziell wirksame Übergang von den bisherigen Fallkostenbudgets der Krankenhäuser zu den nach DRG bemessenen Budgets stattfinden. Am Ende der als Konvergenzphase bezeichneten Jahre 2005 und 2006 soll die Preishöhe landesweit vereinheitlicht sein und die bundesweite Leistungskalkulation feststehen. 
Die Festlegung der Preishöhe, ebenso wie die Krankenhausplanung, bleibt eine Kompetenz der Bundesländer. Auch sind die Länder befugt, Zuschläge zur Sicherung der bedarfsgerechten und wohnortnahen Versorgung der Patienten zu bestimmen. Zuschläge für medizinische Innovationen und Ausbildung fallen ebenfalls unter die Länderkompetenz.

Es ist zu begrüssen, dass das Gesetz die Sicherung der bedarfsgerechten Versorgung den Ländern unterstellt. Die Zuschlagszahlungen, die dem Land ermöglichen, der länderspezifischen Infrastruktur und ihren Bedürfnissen gerecht $\mathrm{zu}$ werden, sind jedoch eingeschränkt durch den gesetzlich vorgeschriebenen Grundsatz der Krankenkassen-Beitragsstabilität. Die Kostenstabilität untergräbt zwangsläufig das Ziel der leistungsbezogenen Vergütung. Bezüglich der Ausbildungszuschüsse ist noch zu klären, ob davon nur universitäre Einrichtungen oder auch kleine Krankenhäuser profitieren. Diese Institutionen leisten einen grossen Beitrag zur ärztlichen Weiterbildung und sollten nicht benachteiligt werden.

Neben der Bundesärztekammer gehören viele andere Verbände zu den Kritikern des straffen Zeitplans, z. B. die Deutsche Krankenhaus Gesellschaft DKG oder der Marburger Bund. Ausser der unsicheren ökonomischen Situation in Folge einer kurzen Anpassungsphase ist der Aufwand zu bedenken, der damit einhergeht. Die dadurch entstehende zeitliche Inanspruchnahme hochqualifizierter Kräfte ohne ersichtliche Dringlichkeit ist problematisch. Die schnelle Einführung birgt zudem das Risiko, am Ende fehlerhafte Kalkulationsgrundlagen und eine unzureichende Systemanpassung vorliegen zu haben.

\section{Qualitätssicherung und Kontrolle}

Die Vergütung ist zukünftig mit einer Verpflichtung zur Qualitätssicherung verbunden. Regelmässige Qualitätsberichte werden obligatorisch. Vertragsparteien und Patienten können jederzeit Einsicht erhalten. Diese Massnahme soll unter anderem ökonomisch begründete, verfrühte Entlassungen verhindern. Die Prüfrechte der medizinischen Dienste der Krankenversicherungen werden verbessert. Die Überprüfung der Aufnahmediagnosen und Abrechnungen ist auch ohne konkreten Verdachtsmoment erlaubt. Für allfällige Streitpunkte ist eine paritätisch zusammengesetzte Schlichtungsstelle vorgesehen.

Das Thema der Qualitätssicherung ist nicht neu. Grundsätzlich ist ein System der Qualitätskontrollen zur Verbesserung der medizinischen
Versorgung zu begrüssen. Wirksam und sinnvoll ist es aber nur dann, wenn der notwendige personelle und finanzielle Aufwand der Erstellung und Auswertung berücksichtigt wird. Der Gesetzgeber hat diesen Aspekt zumindest nicht explizit erwähnt. Er geht offensichtlich wie auch in anderen Bereichen davon aus, dass die erwarteten Einsparungen den Mehraufwand abdecken. Die Bundesärztekammer kritisiert die gesetzliche Verpflichtung zur Qualitätssicherung. Sie hält diese Regelung für kontraproduktiv. Ihrer Meinung nach liegt in einem freiwilligen Verfahren ein grösserer Anreiz, Qualitätssicherung für vermehrte Transparenz und Information der Öffentlichkeit und als Instrument des Wettbewerbs zu nutzen.

Kritisch ist die Zunahme der Kompetenzen der medizinischen Dienste zu betrachten. Weitere, rein ökonomisch fundierte Einmischung in die ärztlichen und pflegerischen Entscheidungen ist vorprogrammiert. Durch das Recht der unbegründeten Stichprobenkontrolle entsteht zudem ein Klima des Misstrauens.

\section{Belastungen des Krankenhauspersonals}

Zum Abbau der Belastungen des Krankenhauspersonals ist ein zweckgebundenes Finanzierungsvolumen von 100 Millionen Euro im Jahre 2003 vorgesehen. Die gleiche Summe steht den Krankenhäusern im Jahre 2004 zu, vorausgesetzt, dass sie als Einsparung erwirtschaftet wird. Eine Neuordnung der Arbeitszeitgestaltung zur Reduktion von Bereitschaftsdiensten und Überstunden soll unter anderem damit ermöglicht werden.

Der personelle Ist-Zustand ist ein Mangelzustand. Etwa 15000 ärztliche Stellen werden benötigt und nach Berechnungen der deutschen Dienstleistungsgewerkschaft Ver.di weitere 20000 Schwestern und Pfleger. Die Bundesärztekammer sowie andere ärztliche Vereinigungen verlangen eine längst überfällige Stellenaufstockung und die Umsetzung des europäischen Gerichtsurteils vom 30. Oktober 2000, nach dessen Beurteilung Bereitschaftszeit als Arbeitszeit $\mathrm{zu}$ werten ist. Eine alleinige Neuordnung der Arbeitszeiten wird den Personalmangel nicht beheben. Das 100-Mio.-Euro-Zückerchen des Gesetzgebers ist allenfalls ausreichend, um temporäre Mehrarbeit im Zuge der Systemanpassung zu kompensieren. Die erwarteten Ersparnisse der Krankenhäuser werden weder die neue Mehrarbeit, z.B. durch mehr bürokratische Aufgaben und Qualitätssicherung, noch die bisherige decken. Sollten die aktuellen Personalkosten als Kalkulationsbasis der DRG dienen, werden die 
künftigen DRG-Erlöse den notwendigen Personalbedarf und moderne, medizinische Qualitätsstandards nicht gewährleisten.

\section{Ein persönliches Fazit}

Der Weg zu einer leistungsgerechten Vergütung hat noch einige Hürden zu nehmen. Das hohe Ziel, ein qualitativ hochwertiges, wirtschaftliches und noch dazu leistungsgerechtes System $\mathrm{zu}$ erarbeiten, hat der Gesetzgeber mit dieser Reform nicht erreicht. Leistungsgerechtigkeit ist unter Beibehaltung der Kostendeckung nicht machbar. Höhere Fallzahlen führen zwangsläufig zu einem Preisverfall der Fallpauschalen. Wer mehr leistet, wird bestraft! Das politische Versprechen der Beitragsstabilität ist hinsichtlich der Zunahme der geriatrischen und pflegerischen Aufgaben des stationären Gesundheitswesens ohnehin gewagt. Die demographische
Entwicklung wird unweigerlich zu einer Kostensteigerung führen. Kritisch ist auch die Kostenverlagerung vom stationären auf den ambulanten und privaten Bereich. Kürzere Krankenhausaufenthalte führen unweigerlich zu einer höheren Inanspruchnahme der ambulanten Dienste. Eine entsprechende Aufstockung der ambulanten Mittel ist jedoch nicht erfolgt. Die weitgehende Negierung des Personalmangels im stationären Gesundheitswesen ist nicht annehmbar, weder für die medizinischen Fachkräfte noch für die Patientenschaft, deren Versorgung zwangsläufig darunter leidet. Als letzten Punkt kann ich auch die weitere Zementierung des Kostendrucks als Entscheidungsparameter ärztlichen Handelns nicht gutheissen. Keine Frage, dass Wirtschaftlichkeit gefördert werden muss und gewisse Einsparungen möglich sind, nur darf ein Patient nicht zu einer reinen Kostengrösse verkommen.

Diesen Weg haben wir hoffentlich nicht eingeschlagen. 